\title{
Detection of Wampee Damage based on Hyperspectral Imaging Technology
}

\author{
Wen-Wu Qiu ${ }^{1}$, Wei-Qiang Su${ }^{1}$, Zhao-Yan Cai ${ }^{1}$, Long Dong ${ }^{1}$, Chang-Bao Li ${ }^{2}$, Wei-Kuan Fang ${ }^{1}$, Ye-Qiang Liu ${ }^{1}$, Xiao-Mei \\ Wang $^{1}$, Zhang-Bao Huang ${ }^{1}$, Jian Qiao ${ }^{3 *}$ \\ ${ }^{1}$ Horticulture Research Institute, Guangxi Academy of Agricultural Sciences, Nanning, Guangxi, 530007, China \\ ${ }^{2}$ Agro-food Science and Technology Research Institute, Guangxi Academy of Agricultural Sciences, Nanning, Guangxi, 530007, \\ China \\ ${ }^{3}$ Key laboratory of Tropical Fruit Biology, Ministry of Agriculture and Rural Affairs, South Subtropical Crop Research Institute, \\ Chinese Academy of Tropical Agricultural Sciences, Zhanjiang, Guangdong, 524091, China
}

\begin{abstract}
Wampee is one of the characteristic fruits in southern China, and its brittle and thin skin can easily be damaged. In this study, principal components analysis (PCA) and minimum noise fraction (MNF) analysis were carried out on the two wampee varieties by hyperspectral imaging technology, and $680 \mathrm{~nm}$ was determined to be the optimal characteristic wavelength. The accurate recognition rate obtained from PCA algorithm for wampee samples of two varieties was about $83.75 \%$, and that obtained from MNF algorithm for two variety samples was $85 \%$. It was indicated that the wampee damaged can be identified more accurately and effectively by MNF based on hyperspectral imaging technology
\end{abstract}

\section{Introduction}

Hyperspectral imaging technology, originated in the early 1990s, is a photoelectric detection technology integrated with electronics and optics. It has many advantages of many disciplines and is widely used in the detection of forest plants and agricultural products. Hyperspectral imaging technology is a combination of image technology and hyperspectral technology. When collecting data, spectral image and spectral information can be obtained at the same time. Through a threedimensional data body, data at any point on the image can be obtained, and data information of each spectrum can be obtained. At the same time, it contains spectral and image information, which has the characteristics of high resolution and many wavebands. In addition to the external features such as surface shape, texture, size, etc., it can also reflect the internal information such as the chemical composition of the surface features through Hyperspectral Information. In recent years, it has been more and more used in nondestructive testing of agricultural product quality [2-5].

Hyperspectral imaging technology is mainly divided into spaceborne hyperspectral imaging technology, airborne hyperspectral imaging technology and ground hyperspectral imaging technology. Ground hyperspectral imaging technology has great advantages in agronomic parameter monitoring, such as low cost, easy to carry, high spatial resolution, high spectral resolution and fixed-point monitoring of crop nutrient status and growth.

In the process of fruit collection, due to the poor accuracy and low efficiency of manual sorting for minor damage of fruit, it is difficult to achieve the consistency of classification [1]. Although the application of hyperspectral imaging technology in fruit damage detection in China is relatively late, with the joint efforts of many scientists in our country, great progress has been made in fruit damage and quality identification and detection. a lot of achievements have been made and have been widely used. Baranowski et al. [6] used hyperspectral images in the range of visible light / near infrared $(400 \sim 1000 \mathrm{~nm})$ and short wave near infrared $(1000 \sim 2500 \mathrm{~nm})$, combined with a variety of supervised classification models, to detect apples within 2 weeks of damage. The results show that the hyperspectral imaging technique for damage days detection has good applicability in both spectral ranges. Sun et al. [8] collected hyperspectral images in the range of 400 1000nm, selected six characteristic bands $(580,599,650,675,710$ and $970 \mathrm{~nm})$ using successive projection algorithm, (SPA), and used partial leastsquares-discriminant regression analysis (PLS-DA), support vector machine (SVM) and artificial neural network (ANN) models for modeling and analysis. The system can classify the frostbite grade of peach. Liu et al. [9] extracted three characteristic wavelengths $(681,867$ and $942 \mathrm{~nm}$ ) by quadratic continuous projection algorithm, and then classified them by linear discriminant analysis, support vector machine and back propagation artificial neural network (BP-ANN) model, respectively, and completed the classification and detection of Hanfu apple diseases. Chen et al [11] used hyperspectral imaging technology to collect normal and damaged spectral images of 60 Korla pears in the

\footnotetext{
* Corresponding author: qiaojian.1987@163.com
} 
$380 \mathrm{~nm}-1030 \mathrm{~nm}$ band, selected the characteristic wavelength and reduced the noise of the spectral image, and modeled the spectral image according to the second derivative. Based on this model algorithm, the identification accuracy of normal and damage samples is $93.75 \%$, indicating that it is feasible to identify the damage of Korla pear based on hyperspectral imaging technology. Sun et al. [12] obtained the characteristic wavelength of jujube through different modeling algorithms, and identified the damage area under the SVM classifier. Between the characteristic wavelength $1353 \mathrm{~nm}$ and $1691 \mathrm{~nm}$, the correct recognition rate of jujube damage is about $81.8 \%$, which indicates hyperspectral imaging technology can be used to effectively identify the damage of jujube. Keresztes et al. [13] detected five bruised apples with different degrees of damage by hyperspectral imager, and the prediction accuracy of bruised apples reached $92.4 \%$ using the database template established by region normalization. The results show that the damage degree detection of fruit can be accurately identified by regional normalization, which can provide a theoretical basis for fruit hyperspectral imaging detection in the future. Zhang and $\mathrm{Li}$ [10] uses hyperspectral imaging technology $(400 \sim 1000 \mathrm{~nm})$ to achive rapid identification and non-destructive testing of slight damage of apple, and the recognition rate is $96.67 \%$ based on RELIEFextreme learning machine (Re-ELM) model of characteristic band. a hyperspectral detection algorithm for slight damage of apple is proposed, and the algorithm is used for independent component analysis (independentcomponentanalysis,ICA) transform according to the characteristic band. The third component image of ICA is selected for adaptive threshold segmentation to obtain the damaged image. The detection of all hyperspectral images shows that the final recognition rate of the algorithm is more than $94 \%$, indicating that the algorithm can effectively identify the damaged areas of apples. It can be seen that the effective identification of fruit damage can be achieved by using hyperspectral imaging technology through appropriate algorithm selection and modeling processing for different kinds of fruit.

Clausenalansium (Lour.) Skeels, also known as spotted yellow peel, fruit yellow, yellow marble, yellow fade, yellow LOX, yellow orange, yellow peel, yellow jar, jade jar, yellow bark, yellow sandalwood, golden marble, Wang marble, Huang Dan, Huang Duan and Haimei, the English name Chinese Wanpee, Puactate Wampee, Wampee, Wampi, is the evergreen tree of the genus Rutaceae. It is native to South and Southwest China and has been cultivated for more than 1500 years [6]. The fruit is Mainly produced in Guangdong, Guangxi, Yunnan, Sichuan, Hainan and other places. The flesh of yellow fruit is thin, the fruit taste is sweet, it is one of the summer fruits in the south, and its seeds are also known as wampee kernels. Yellow fruit has a slight gas, taste pungent, sweet, sour, lukewarm, slightly bitter. It has the effect of invigorating qi, eliminating food and resolving phlegm. It is mainly used for food accumulation and fullness, epigastric pain, hernia pain, phlegm, cough and asthma.

In the past years, with the continuous development of society, there is a huge demand for fruit in domestic and foreign markets, but there is very little in our country for export. The main reason for this situation is that western countries have formulated increasingly strict agricultural standards and put forward high requirements for safety, green and high quality of agricultural products. At present, there is a big gap in the standardization system of fruit production between China and the western countries. The main reason is that the testing methods of our country lag far behind the western countries, which makes it difficult for the export products to meet the agricultural standards of the western countries. Therefore, this paper intends to carry out accurate and rapid nondestructive testing technology of wampee damage based on hyperspectral imaging technology, in order to provide theoretical basis and practice for the non-destructive quality analysis of yellow peel and other fruits, and has important theoretical significance and practical value for improving the international status and competitiveness of Chinese fruits and promoting the revitalization of rural industries.

\section{Materials and methods}

\subsection{Experiment materials}

The yellow peel fruits used in this experiment are the warieties of "Guifei" and "Jixin", which are purchased from the supermarket in Zhanjiang city. 40 wampee fruits of similar size are carefully selected from each variety, and 20 of the each varieties are randomly selected for injury treatment.

\subsection{Experimental instrument}

SOC710VP portable infrared imager, Y-252\5000 multi-function mobile power supply, two $75 \mathrm{~W}$ halogen lamps, sample loading station.

\subsection{Image correction}

Spectral imaging system may be disturbed by the current of electronic equipment in the process of collecting hyperspectral image, resulting in a series of dark current and noise, which affects the resolution and band of spectral image and then the brightness of each band will be different. Therefore, in order to weaken the light influence of the hyperspectral imaging image and reduce the noise, it is necessary to correct the spectral reflectivity formula, and the correction image R (Fig. 1) can be obtained by using the formula[14].

$$
\mathrm{R}=(\mathrm{U}-\mathrm{B}) /(\mathrm{W}-\mathrm{B})
$$




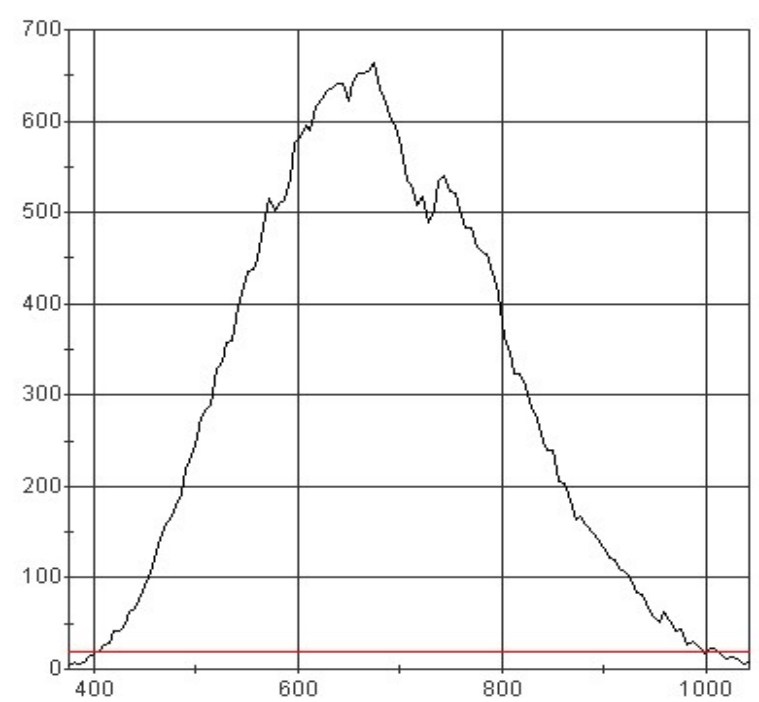

Original hyperspectral image

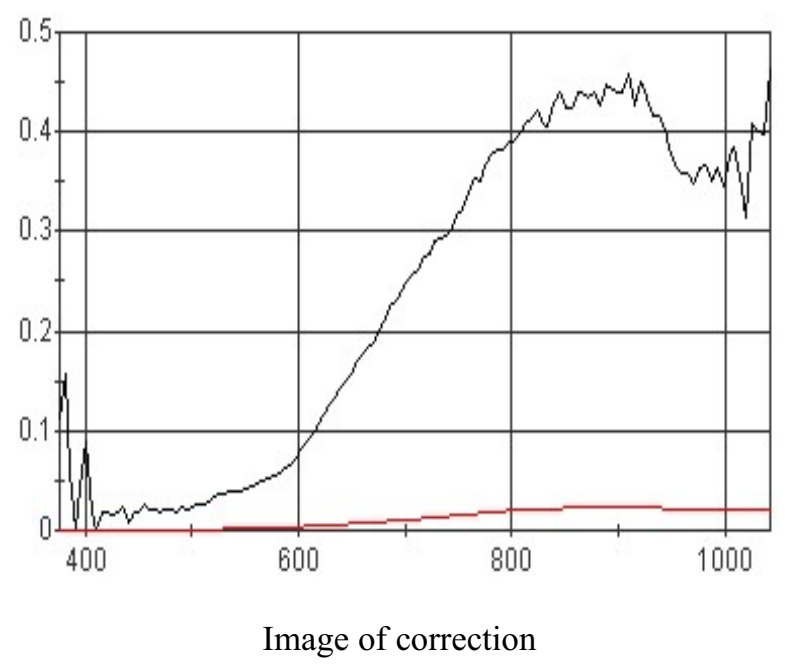

Fig.1 Hyperspectral image of original and correction

Among them: $\mathrm{R}$ represents the hyperspectral image corrected by the reflectivity formula; $U$ represents the most original hyperspectral image; B represents the dark spectral image obtained by the spectral lens after turning off the light source and screwing the lid on; $\mathrm{W}$ represents the spectral image that is first put into the reference version.

\subsection{Data processing}

Image processing are mainly based on HyperScanner 2.0, SRAnal 710e, ENVI 5.3 (64-bit), ENVI Classic (64-bit) and other software.

\section{Results and analysis}

\section{1 the spectral image acquisition conditions}

Based on the results of comparison of many experiments, it was found that the parameters of best hyperspectral image was obtained, and the spectral resolution was 5.6,

the integration time was $22 \mathrm{~ms}$, and the height of the hyperspectral imager was $28 \mathrm{~cm}$.

\subsection{Reflectance spectra of normal and injured areas of wampee}

When spectral characteristics of normal and injured regions of wampee were studied, it is necessary to collect spectral data of region of interesting (ROI) in normal and injured regions. Each wampee image has characteristic information under different wavelength conditions. Fig. 2 shows the spectral curves of normal and damaged regions in the range of $360 \mathrm{~nm}-1050 \mathrm{~nm}$ bands. In the picture, three curves are the spectral curves of the normal region, and three are the spectral curves of the damaged area of the wampee.

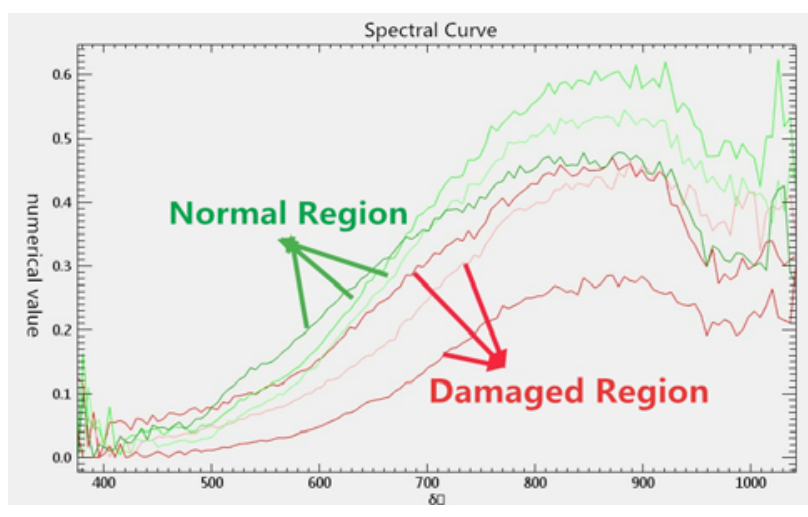

Y axis: spectral reflectivity $\mathrm{X}$ axis: wavelength (nm)

Fig. 2. Spectral curves of normal and damaged regions of wampee (Note: red lines represent spectral curves of damaged areas, green lines represent spectral curves of normal areas)

It can be seen from Fig. 2 that the reflection spectrum values of the normal and damaged regions are relatively close when the wave band value is below $600 \mathrm{~nm}$. Below the wavelength of $375 \mathrm{~nm}$ and above $1000 \mathrm{~nm}$, the spectral curves in these two bands contain a large number of unstable characteristics and noise, so we should select the hyperspectral data in the range of $375 \mathrm{~nm}-1000 \mathrm{~nm}$ for analysis and processing when dealing with the data, and the spectral curve of the normal region in the $600 \mathrm{~nm}-1000 \mathrm{~nm}$ band is higher than that in the damaged region.

\subsection{The selection of characteristic wavelength of hyperspectral image}

\subsubsection{PCA principal component analysis}

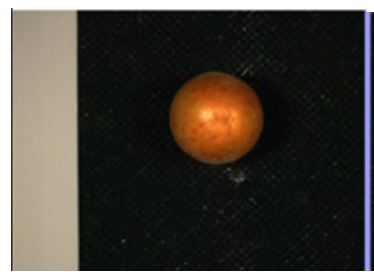

a

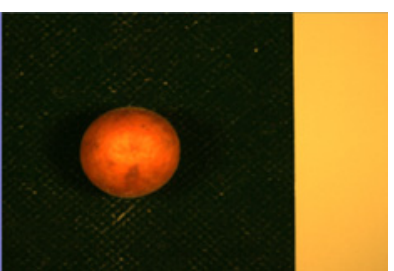

b 


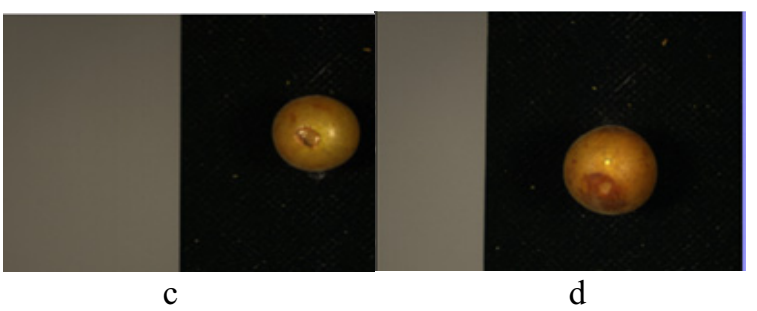

Fig. 3.Original image of damaged wampee (a, b, c, d represent fruit samples of two varieties, a and b are "Guifei" variety, c and d are "Jixin" variety, the same below)

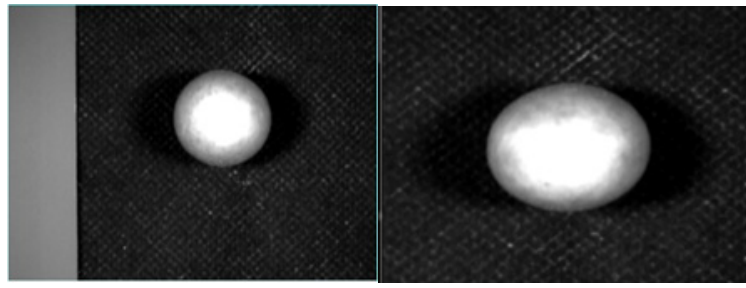

a

b

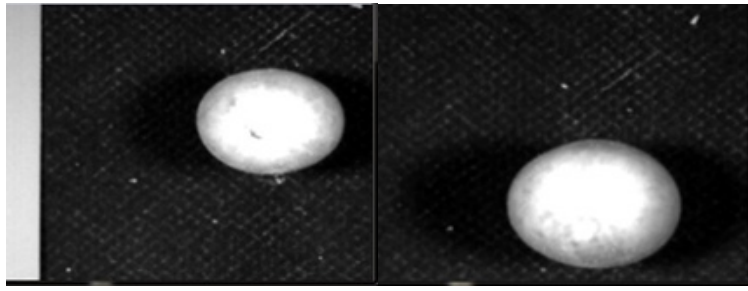

a

b

Fig.4. PC1 of damaged wampee
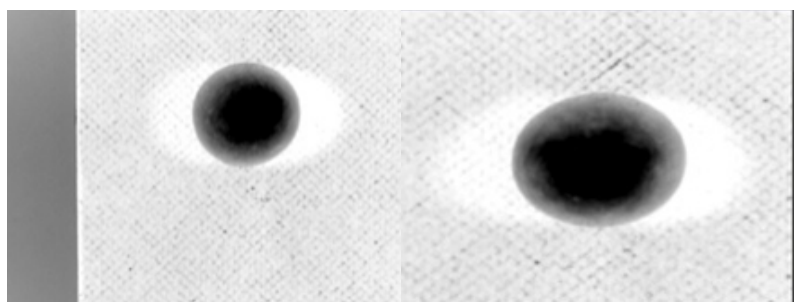

a

b
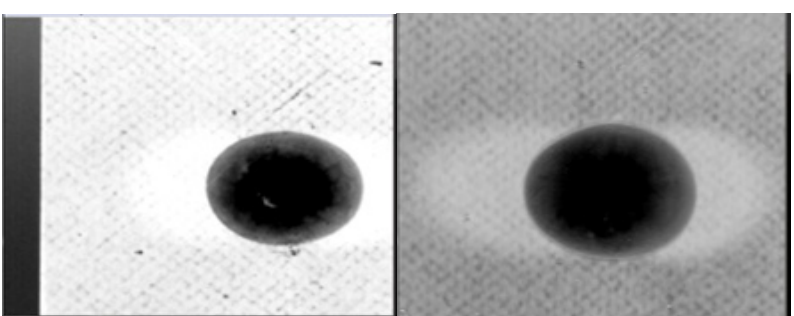

c

d

Fig.5. PC2 of damaged wampee

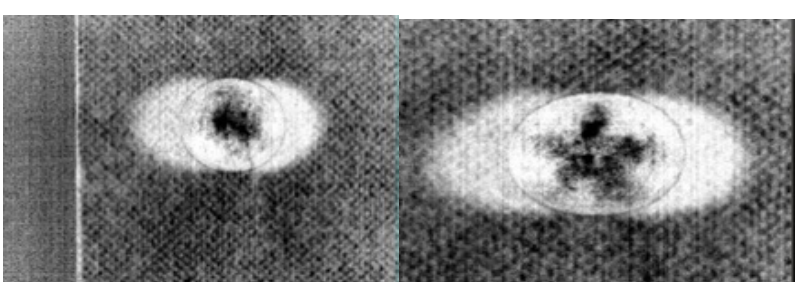

a

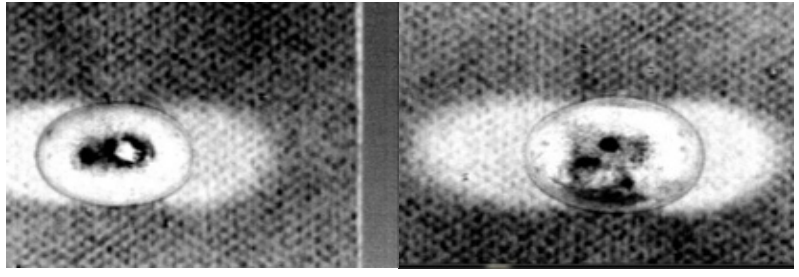

$\mathrm{c}$

d

Fig.6. PC3 of damaged wampee

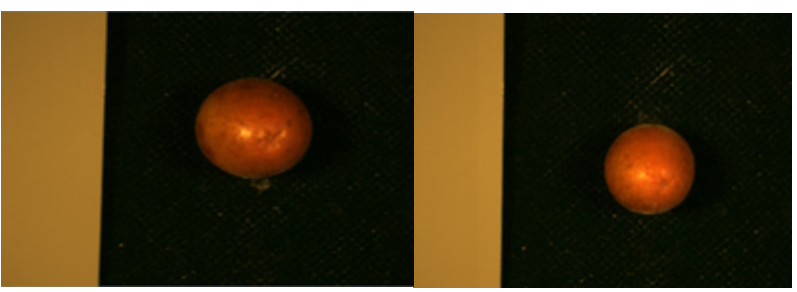

a

b

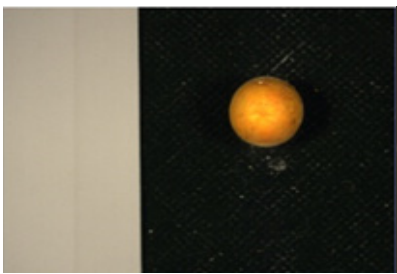

c

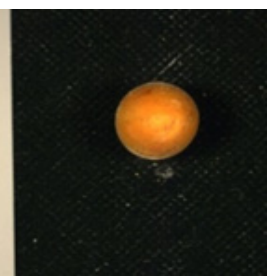

d
Fig. 7. original image of normal wampee

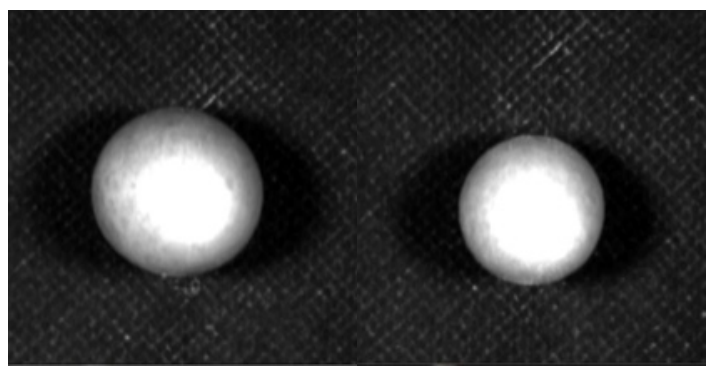

a

b

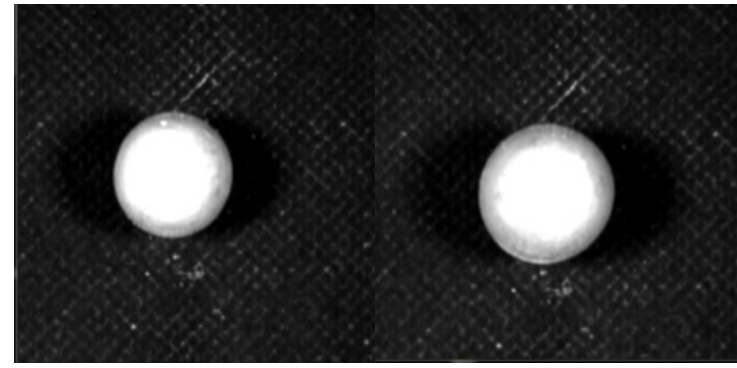

d

Fig. 8. PC1 of normal wampee 


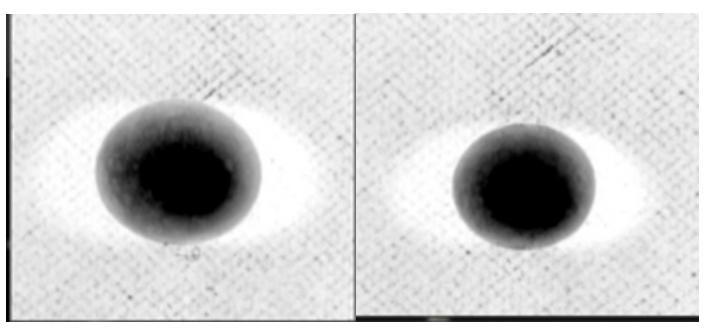

a

$\mathrm{b}$

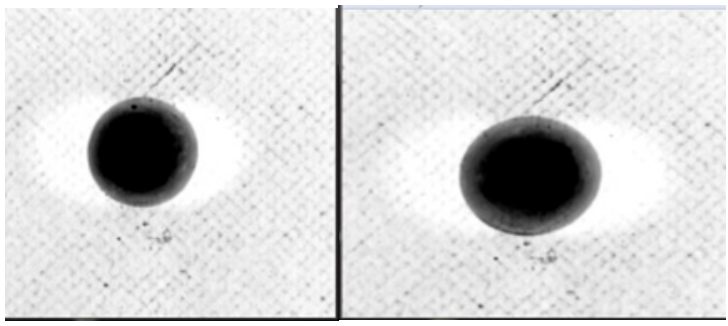

C

d

Fig. 9. PC2 of normal wampee

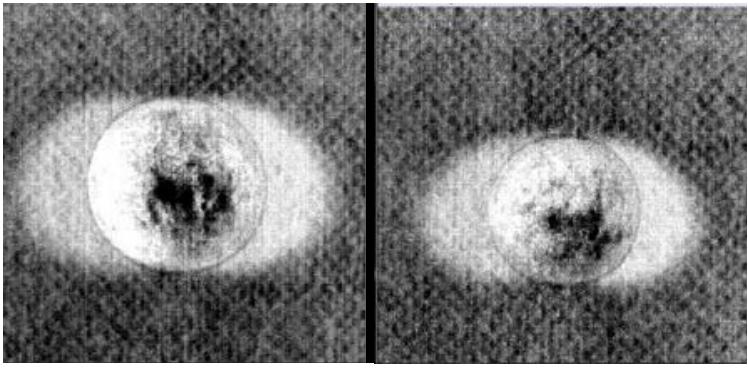

b

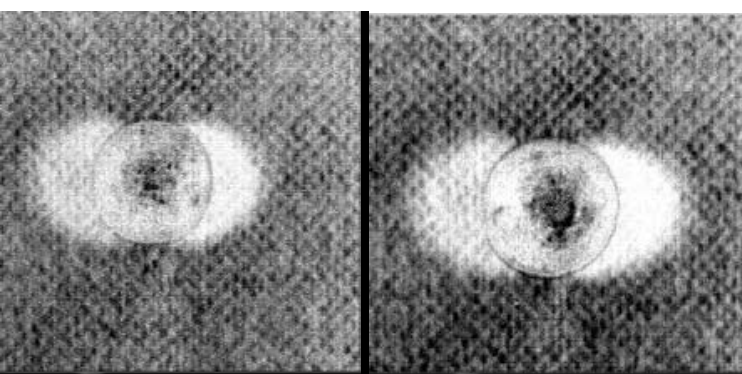

d

Fig. 10. PC3 of normal wampee

\begin{tabular}{rrr}
\hline PC & Ei genvalue & Percent \\
1 & 0.5256 & 75.378 \\
2 & 0.1466 & 96.408 \\
3 & 0.0034 & 96.888 \\
4 & 0.0030 & 97.318 \\
5 & 0.0028 & 97.718 \\
6 & 0.0024 & 98.068 \\
7 & 0.0022 & 98.388 \\
8 & 0.0014 & 98.598 \\
9 & 0.0012 & 98.758 \\
10 & 0.0009 & 98.898 \\
11 & 0.0009 & 99.018 \\
12 & 0.0007 & 99.118 \\
13 & 0.0006 & 99.208 \\
14 & 0.0005 & 99.278 \\
15 & 0.0005 & 99.348 \\
16 & 0.0004 & 99.398 \\
17 & 0.0003 & 99.448 \\
18 & 0.0003 & 99.498 \\
19 & 0.0003 & 99.538 \\
20 & 0.0002 & 99.568 \\
21 & 0.0002 & 99.608 \\
22 & 0.0002 & 99.638
\end{tabular}

Fig. 11. PCA principal component feature information

PCA principal component analysis is the most commonly used method and the most important operation method to process the hyperspectral data with the ENVI software. As can be seen from Fig. 11, the information is mainly in the first three principal components based on the PCA, so we only need to save the three PCA principal component information. We get the three principal components PC1, PC2 and PC3 after PCA principal component analysis of the original image. By comparing Fig. 3- Fig. 6 and Fig. 7- Fig. 10, it can be found that the third principal component PC3 is the best image for detecting wampee damage

\subsubsection{Analysis of MNF}

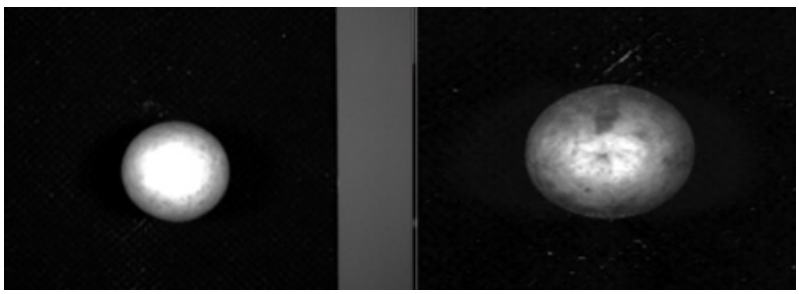

a

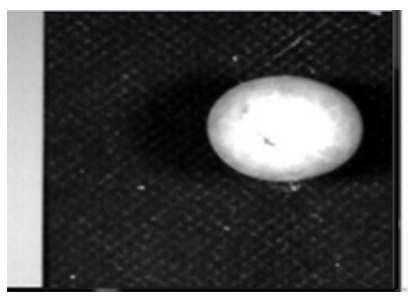

C b

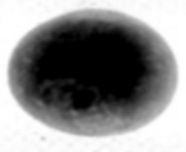

d

Fig.12. MNF1 of damaged wampee 


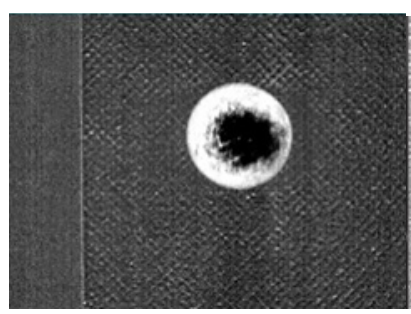

a

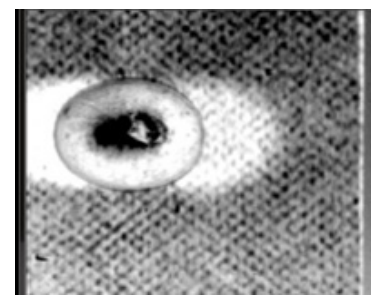

c

Fig.13. MNF3 of damaged wampee

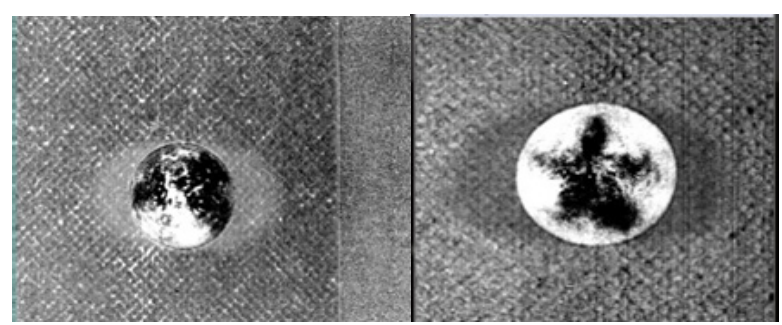

b

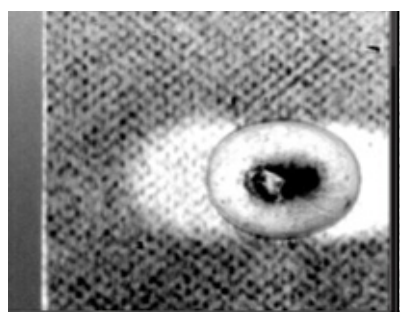

c

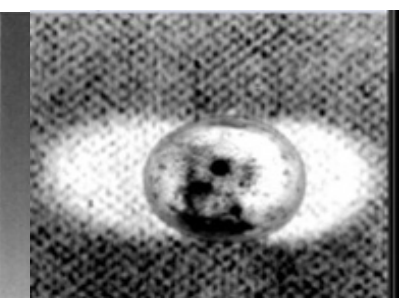

d

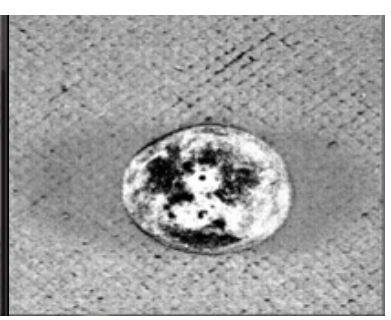

d

Fig.14. MNF1 of damaged wampee

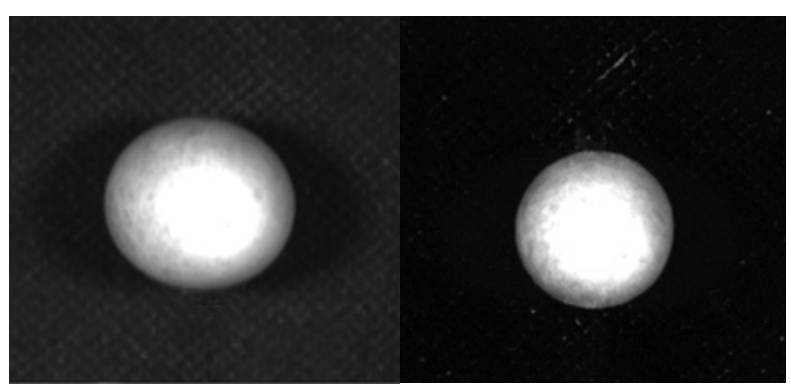

a

b

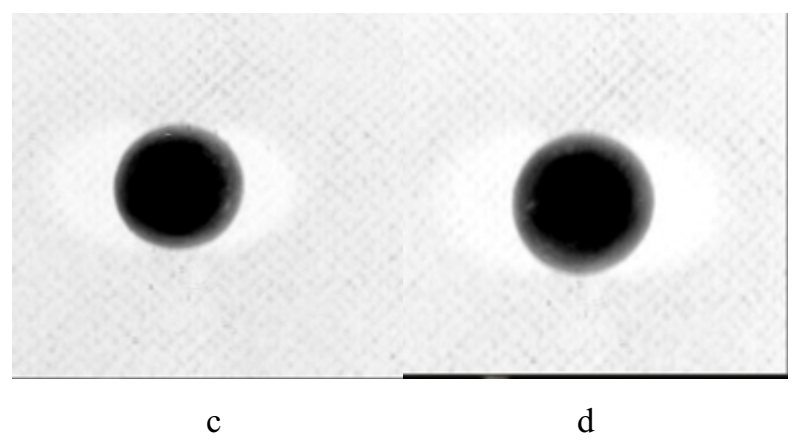

Fig.15. MNF1 of normal wampee

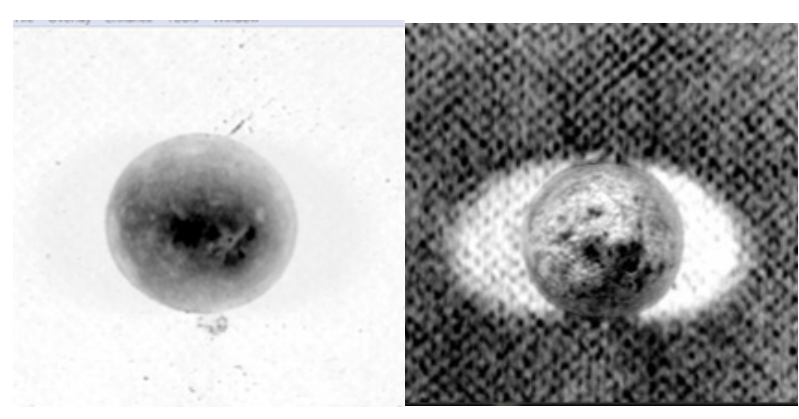

a

b

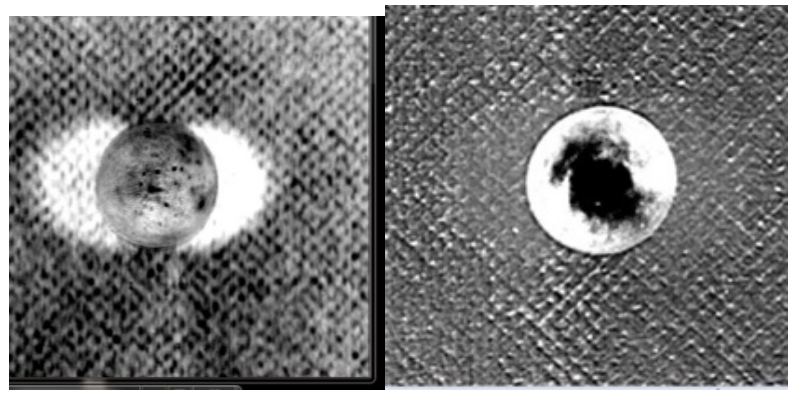

c

d

Fig.16. MNF2 of normal wampee
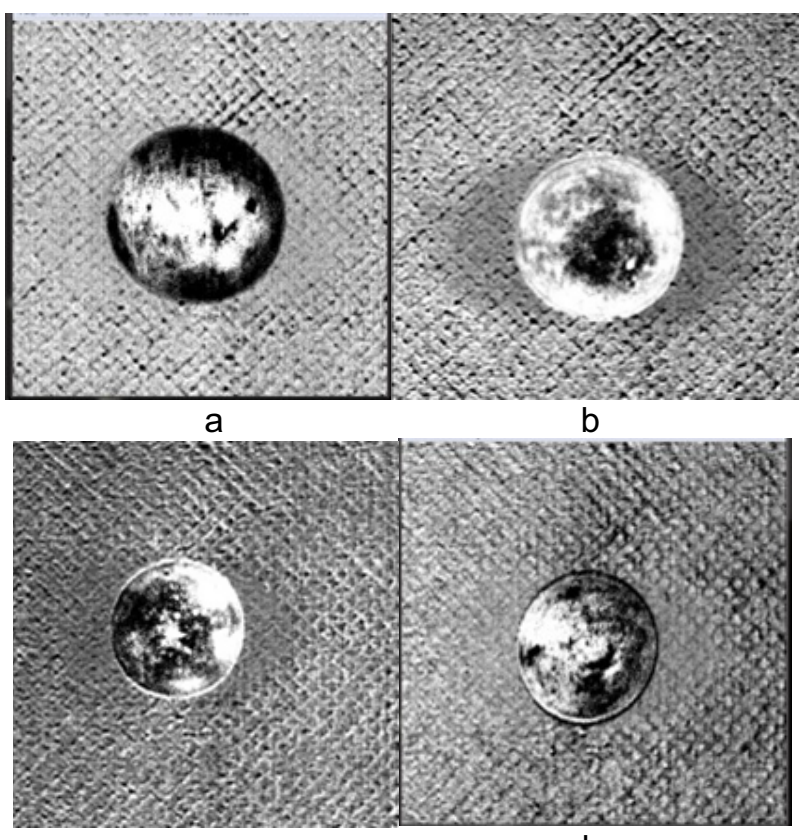

C

Fig.17. MNF3 of normal wampee 


$\begin{array}{rrr}\text { MMF } & \text { Ei genvalue } & \text { Percent } \\ 1 & 1014.9438 & 51.658 \\ 2 & 761.2456 & 90.398 \\ 3 & 36.8036 & 92.268 \\ 4 & 16.1158 & 93.088 \\ 5 & 5.0184 & 93.348 \\ 6 & 2.9823 & 93.498 \\ 7 & 2.2023 & 93.608 \\ 8 & 1.8039 & 93.698 \\ 9 & 1.5059 & 93.778 \\ 10 & 1.3548 & 93.848 \\ 11 & 1.3131 & 93.918 \\ 12 & 1.2970 & 93.978 \\ 13 & 1.2260 & 94.048 \\ 14 & 1.2006 & 94.108 \\ 15 & 1.1616 & 94.168 \\ 16 & 1.1422 & 94.218 \\ 17 & 1.1208 & 94.278 \\ 18 & 1.1067 & 94.338 \\ 19 & 1.0955 & 94.388 \\ 20 & 1.0725 & 94.448 \\ 21 & 1.0691 & 94.498 \\ 22 & 1.0605 & 94.558 \\ 1 & & \end{array}$

Fig.18. feature information of MNF

As can be seen from Fig. 18, in the full-band MNF transformation of hyperspectral images, the characteristic information of the image is mainly in the first three, we only need to retain the first three MNF characteristic bands, and analyze these $3 \mathrm{MNF}$ characteristic bands. From Fig. 12 to Fig. 17, although the content of the image response of each component is the same, it can be seen that the damage was found clearly from MNF3, which was obtained from the comparison of the MNF transform image. Because of the effectiveness of the characteristic band, the MNF3 can represent the whole band with MNF transform processing and can be used for accurate damage identification of fruits.

\subsubsection{Band information results and correlation coefficient analysis}

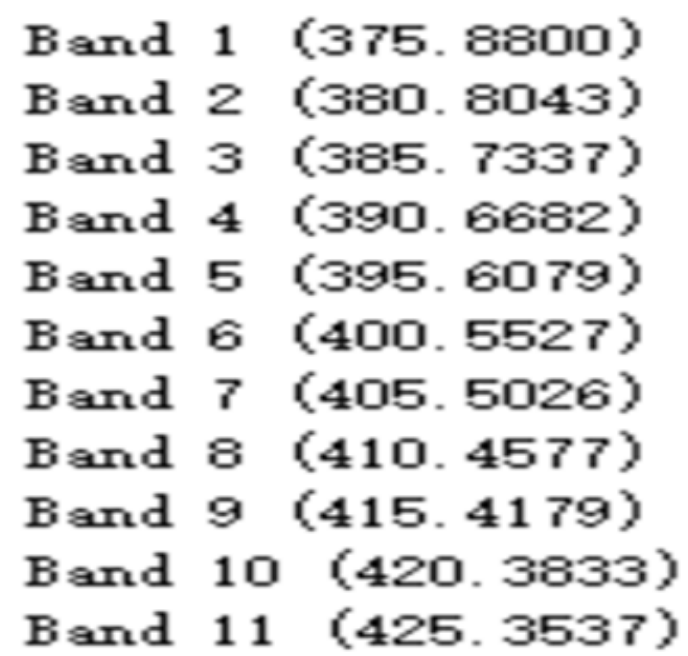

Fig. 19. the corresponding wavelengths of each band (the wavelength increases by $5 \mathrm{~nm}$ with each additional band)

It can be seen from Fig. 11 and Fig. 18 that the main function of PCA is to concentrate the information on the first three bands, and we only need to pay more attention on the first three, namely Band1, Band2, and Band3. Through Fig. 4- Fig. 6 and Fig. 12- Fig. 14, it can be found that the third principal component has the best recognition effect, so the third principal component, Band3, can represent the characteristic band of wampee damage. The wavelength corresponding to the maximum weight coefficient of each principal component image is the best characteristic wavelength, and the weight coefficient is the eigenvector in the correlation coefficient matrix. Generally, the first column is the weight coefficient of the first principal component [15]. Accordingly, the eigenvector of the third principal component, Band3, is shown in Table 1, and the wavelength corresponding to the largest eigenvector is $680 \mathrm{~nm}$, which is the best characteristic wavelength.

Table 1. maximum contribution value (eigenvector)

\begin{tabular}{|c|c|c|c|c|c|c|}
\hline Eigenvector & $\begin{array}{c}\text { Band 1 } \\
(375 \mathrm{~nm})\end{array}$ & $\begin{array}{c}\text { Band 2 } \\
(380 \mathrm{~nm})\end{array}$ & $\begin{array}{c}\text { Band 3 } \\
(385 \mathrm{~nm})\end{array}$ & $\begin{array}{c}\text { Band 61 } \\
(675 \mathrm{~nm})\end{array}$ & $\begin{array}{c}\text { Band 62 } \\
(680 \mathrm{~nm})\end{array}$ & $\begin{array}{c}\text { Band 63 } \\
(685 \mathrm{~nm})\end{array}$ \\
\hline Band 1 & -0.004099 & 0.00003 & 0.00073 & -0.073279 & -0.080432 & -0.080929 \\
\hline Band 2 & 0.0123910 & 0.020391 & 0.033361 & 0.060252 & 0.052800 & 0.044773 \\
\hline Band 3 & 0.019438 & 0.023696 & 0.031125 & 0.111044 & 0.111118 & 0.111114 \\
\hline
\end{tabular}

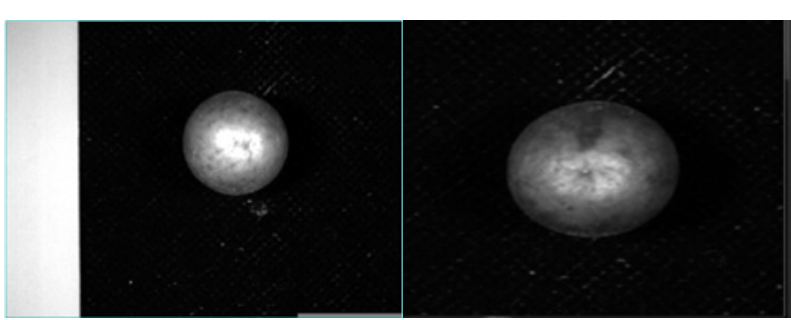

a b

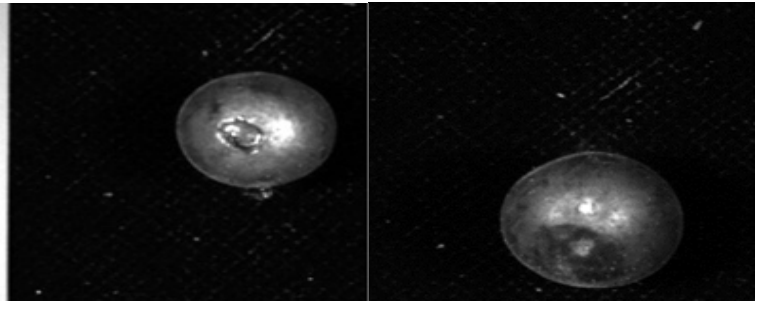

$\mathrm{c}$

d

Fig. 20. image of damaged wampee under characteristic wavelength of $680 \mathrm{~nm}$ 


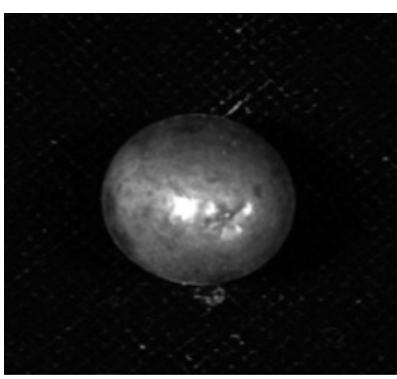

$\mathrm{a}$

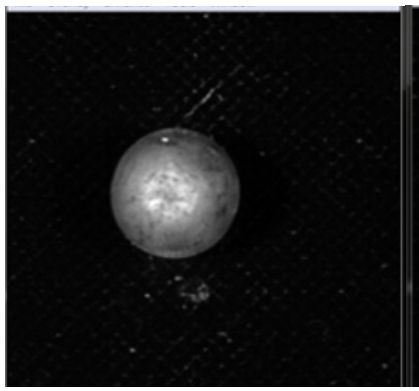

c

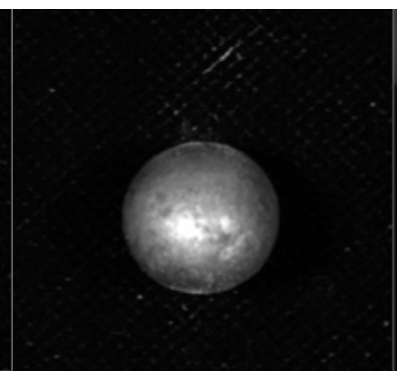

b

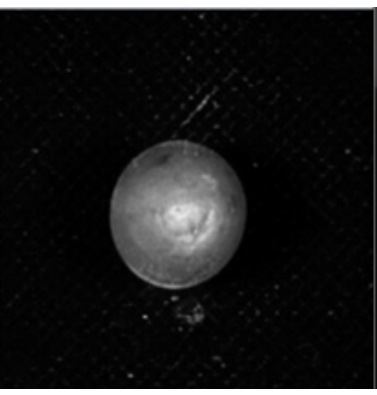

d
Fig. 21. image of normal wampee under characteristic wavelength of $680 \mathrm{~nm}$

\subsection{Damage detection results of wampee}

Hyperspectral detection and image processing were carried out on 40 samples of "Guifei" variety and 40 samples of "Jixin" variety. The results of reflection spectra are shown in Table 2. The average correct recognition rate of the reflection spectrum of each wampee sample is about $60 \%$, mainly because the skin of the wampee is incomplete, there is some slight damage, and because of the illumination in the process of collecting hyperspectral data images, which will cause a great error in the reflection spectrum.

PCA principal component analysis was carried out on the damaged wampee of the two varieties. The results are shown in Table 3. The damage recognition rate can reach $83.75 \%$. The reason why the normal samples can not be correctly identified may be that the reflection of the light source causes errors in the process of spectral collection. At the same time, the yellow fruit has a core, and the spectral image can not be obtained correctly when the core and the damaged area overlap.

Using MNF transform analysis to identify the damaged wampee, the results are shown in Table 4, the correct recognition rate can reach $85 \%$, but the correct recognition rate of normal wampee is still low, the main reason is the light reflection of the yellow peel, at the same time, the brittle and thin of skin in wampee is easy to cause slight abrasions, which has a certain impact on the final results.

Table 2. results of reflection spectrum

\begin{tabular}{|c|c|c|c|c|}
\hline \multicolumn{2}{|c|}{ Sample type } & Number & Correct detection number & Correct rate \\
\hline \multirow{2}{*}{ Guifei } & Normal & 20 & 15 & $75 \%$ \\
\cline { 3 - 5 } & Damaged & 20 & 16 & $80 \%$ \\
\hline Jixin & Normal & 20 & 14 & $70 \%$ \\
\hline & Damaged & 20 & 14 & $70 \%$ \\
\hline Total & & & & $73.75 \%$ \\
\hline
\end{tabular}

Table 3. the detection result of PCA

\begin{tabular}{|c|c|c|c|c|}
\hline \multicolumn{2}{|c|}{ Sample type } & Number & Correct detection number & Correct rate \\
\hline \multirow{3}{*}{ Guifei } & Normal & 20 & 16 & $80 \%$ \\
\cline { 2 - 5 } & Damaged & 20 & 18 & $90 \%$ \\
\hline \multirow{3}{*}{ Jixin } & Normal & 20 & 14 & $70 \%$ \\
\cline { 2 - 5 } & Damaged & 20 & 19 & $95 \%$ \\
\hline Total & & & & $83.75 \%$ \\
\hline
\end{tabular}

Table 4. the detection result of MNF

\begin{tabular}{|c|c|c|c|c|}
\hline \multicolumn{2}{|c|}{ Sample type } & Number & Correct detection number & Correct rate \\
\hline \multirow{3}{*}{ Guifei } & Normal & 20 & 13 & $76 \%$ \\
\cline { 2 - 5 } & Damaged & 20 & 20 & $100 \%$ \\
\hline \multirow{3}{*}{ Jixin } & Normal & 20 & 15 & $75 \%$ \\
\cline { 2 - 5 } & Damaged & 20 & 20 & $100 \%$ \\
\hline Total & & & & $85 \%$ \\
\hline
\end{tabular}




\section{Discussion and conclusion}

This study is mainly based on the non-destructive testing of damaged wampee based on hyperspectral imaging technology. The reflection spectral image was obtained by the reflection spectral processing of the collected hyperspectral images of normal wampee and damaged wampee. The reflection spectral rate of the normal area of wampee is higher than that of the damaged area, so the fruit of damaged can be preliminarily identified by this.

Based on the PCA analysis and MNF analysis of the image data of normal wampee and damaged wampee, it can be found that the optimal characteristic wavelength is $680 \mathrm{~nm}$, which can be used to detect the damage of wampee instead of the whole wave band. The damage of wampee can be effectively identified through the PCA analysis of the hyperspectral image of $680 \mathrm{~nm}$, and the correct recognition rate can reach about $83.75 \%$. The correct recognition rate of wampee damage can reach $85 \%$ based on MNF analysis, which is the highest accurate solution and the optimal identification be carried out. This study provides a theoretical basis for the rapid analysis of wampee injury by hyperspectral imaging technology.

\section{Acknowledgements}

This study was supported by the grant from the Central Public-interest Scientific Institution Basic Scientific Research Fund for Chinese Academy of Tropical Agricultural Sciences (No. 1630062017009; 1630062017017 ; 1630062020008; 1630062020009); the Fund of Operation of Integrated Laboratory for SSCRI; the Innovation Team of Modern Agricultural Industry Technology System for Rare Fruits in Guangdong Province of China (2019KJ116); the Youth Science and Technology Talent Development Project of Guizhou Province Education Department(Guizhou jiao he KY [2017]322).

\section{Reference}

1. Y.W. Tian, Y. Cheng, X.Q.Wang, et al. Recognition method of insect damage and stem/calyx on apple based on hyperspectral imaging. Transactions of the CSAE, 2015,31(4):325-331. (in Chinese with English abstract)

2. W.Q. Huang, L.P. Chen, J.B. Li, et al. Effective wavelengths determination for detection of slight bruises on apples based on hyperspectral imaging. Transactions of the CSAE,2013,29(1):272-277. (in Chinese with English abstract)

3. S.X. Cheng, Y.N. Shao, D. Wu, et al. Determination of rice leaf blast disease level based on visible-nearinfrared spectroscopy. Journal of Zhejiang University (Agriculture and Life Sciences), 2011,37(3):307-311. (in Chinese with English abstract)
4. W.H. Lee, M.S. Kim, H. Lee, et al. Hyperspectral near-infrared imaging for the detection of physical damages of pear. Journal of Food Engineering, 2014,130:1-7.

5. X.H. Wei, S. Wu, X.D Fan, et al. Identification of slight bruises on winter jujube based on hyperspectral imaging technology. Transactions of the Chinese Society for Agricultural Machinery, 2015,46(3):242-246. (in Chinese with English abstract)

6. W.C. Guo, S.R. Zhou, X.Y. Dong, et al. A brief introduction to the varieties of wampee. Special Economic Animal and Plant. 2018,21(4):49-50

7. P. Baranowski, W. Mazurek, J. Pastuszka-wozniak. Supervised classification of bruised apples with respect to the time after bruising on the basis of hyperspectral imaging data. Postharvest Biology and Technology, 2013,86:249-258.

8. Y. Sun, X.Z. Gu, K. Sun, et al. Hyperspectral reflectance imaging combined with chemometrics and successive projections algorithm for chilling injury classification in peaches. LWT - Food Science and Technology, 2016,75: 557-564.

9. S.J. Liu, Y.W. Tian, F. Zhang, et al. Hyperspectral imaging for nondestructive detection of Hanfu apple diseases using successive projections algorithm and BP neural network. Food Science, 2017,38(8):277282. (inChinese with English abstract)

10. M. Zhang, G.H. Li. Detection method of slight bruises of apples based on hyperspectral imaging and RELIEF-extreme learning machine. Journal of Zhejiang University (agricultural and Life Sciences Edition), 2019,45(1):126-134

11. X.X Chen, C.T. Guo, C. Zhang, et al. Visual Detection Study on Early Bruises of Korla Pear Based on Hyperspectral Imaging Technology [J]. Spectroscopy and Spectral Analysis, 2017, 37(1): 150-155.

12. S.P. Sun, J. Peng, R. Li, et al. Early Detection of Mechanical Damage in Chinese Winter Jujube (Zizyphus jujuba Mill.cv.Dongzao) Using NIR Hyperspectral Images [J].Food Science, 2017(2): 301-303.

13. J.C. Keresztes., E. Diels, M. Goodarzi, et al. Glare based apple sorting and iterative algorithm for bruise region detection using shortwave infrared hyperspectral imaging $[\mathrm{J}]$. Postharvest Biology and Technology, 2017.130:103-115

14. N.Y. Rivera, J. Gomez-Sanchis, J. ChanonaPerez, et al. Early detection of mechanical damage in mango using NIR hyperspectral images and machine learning[J].Biosystems Engineering , 2014, 122(3): 91-98.

15. M.S. Kim, A.M. Lefcourt, K. Chao, et al. Multispectral detection of fecal contamination on apples based on hyperspectral imagery: Part II. Application of hyperspectral fluorescence imaging. Transactions of ASAE, 2002, 45(6):2 039 2047. 